\title{
The Potential of Human-derived Periodontal Ligament Stem Cells to Osteogenic Differentiation: An In vitro Investigation
}

\author{
Behzad Houshmand ${ }^{1}$, Omolbanin Amjadi ${ }^{2}$, Alireza Rafiei ${ }^{2 *}$, Mohammadali Rouzegar ${ }^{3}$, Mohammadreza Abrishami ${ }^{1}$, Mohammadreza \\ Talebi Ardakani ${ }^{1}$ \\ ${ }^{1}$ Department of Periodontics, School of Dentistry, Shahid Beheshti University of Medical Sciences, Tehran, Iran. \\ ${ }^{2}$ Molecular and Cell Biology Research Center, Department of Immunology, School of Medicine, Mazandaran University of Medical Sciences, \\ Mazandaran, Iran. \\ ${ }^{3}$ Department of periodontology, School of Dentistery, Ilam University of Medical Sciences, Ilam, Iran.
}

\section{Received: 28 Aug 2014}

Revised : 21 Sep 2014

Accepted: 5 Oct 2014

Corresponding Authors:

Alireza Rafiei

Molecular and Cell Biology Research

Center, Department of Immunology,

Faculty of Medicine, KM 17

Khazarabad Road, Khazar Sq, Sari,

Iran.

Phone: +98-1133543614

E-mail: rafiei1710@gmail.com

\begin{abstract}
Background: Periodontal ligament stem cells (PDLSCs) are considered as a type of mesenchymal stem cell that is beneficial target for numerous clinical applications in periodontal tissue regeneration therapy.

Materials and Methods: This study examined the effects of dexamethasone (Dex) on human PDLSCs in vitro. PDLSCs obtained from the roots of patient's teeth were cultured with Dex $(0.01 \mu \mathrm{M})$, and their proliferation was measured. The osteogenic differentiation was assessed by alkaline phosphatase (ALP) activity and Alizarin Red-S staining for calcium deposition.

Results: After the administration of $0.01 \mu \mathrm{M}$ Dex, the activity of ALP increased significantly. Furthermore, mineralized nodule formation showing the intracellular calcium deposition was significantly higher in the Dex-treated cells than that of the control cells.

Conclusion: Collectively, Dex has positive effects on osteogenic differentiation of human PDLSCs in vitro. It is suggested that PDLSCs may serve as a potential material for periodontal tissue regeneration.
\end{abstract}

Keywords: Periodontal ligament stem cells; Osteogenic differentiation; Dexamethasone

Please cite this article as: Houshmand B, Amjadi O, Rafiei A, Rouzegar M, Abrishami M, Talebi Ardakani M. The Potential of Human-derived Periodontal Ligament Stem Cells to Osteogenic Differentiation: An In vitro Investigation. Res Mol Med. 2014; 2 (4): 18-23

\begin{abstract}
Introduction
Periodontal disease or periodontitis is a chronic infection associated with different organs consisting of epithelial tissue and soft and mineralized connective tissues, resulting in inconvenience effects of lost tooth and emerging systemic diseases $(1,2)$. This is an inflammatory disease as the result of interaction between subgingival plaque microbiota and host responses (3). It is estimated that this high prevalent disease may affect $90 \%$ of the worldwide population. Periodontal diseases are associated with adverse pregnancy outcomes, coronary heart disease (4), stroke, pulmonary disease, and diabetes (5). Therefore, it seems critical to manage treatment planning. To date, several therapeutic ways, such as implantation of autografts, allografts and alloplastic
\end{abstract}

materials, chemical root conditioning, growth factors, guided tissue regeneration (GTR), and combination of these approaches have been used in clinical practice (6). All therapies aim to reduce further attachment loss, connective tissue damage and therefore improving periodontal regeneration and healing (7). Current therapies is based on the Melcher's hypothesis, "compartmentalization". $\mathrm{He}$ proposed that the connective tissue between tooth and periodontal tissue are categorized into four groups: the lamina propria of the gingiva (gingival corium), the periodontal ligament (PDL), the cementum, and the alveolar bone. These four types of cells have the potential to recreate from their original and undifferentiated cells and repopulate in the wound 
area (8). Therefore, only PDL are the cells that could be repopulated for periodontal regeneration.

PDL is a connective tissue that consists of unmineralized network of collagen fibers (9), and locates between tooth root and alveolar bone (10). It covers the root of the tooth, functionally maintaining teeth in alveolar bone (11) and contributes in periodontal regenerating in response to wounding, periodontitis, and orthodontic tooth movements after treatment. The presence of pluripotent stem cells within PDL make it as a suggestive tissue involving in preserving tissue homeostasis and periodontal regeneration. Periodontal regeneration can occur following the migration of periodontal ligament stem cells (PDLSCs) and their differentiation into cementoblasts, osteoblasts and fibroblasts, and finally develop into extracellular matrix similar to periodontal tissue (12). This ability has been attributed to the pluripotency of PDL-derived stem cells originating from the neural crest and shed new lights on therapeutic approaches (13).

In the current study, stem cells obtained from patient's premolars-derived PDL were investigated for in vitro evaluation of the ability of PDL to differentiate into osteoblasts and calcified deposits formation. For this purpose, morphological features and calcified nodule arrangement were monitored under selective cultivation conditions.

\section{Materials and Methods \\ Patient selection}

PDLSCs were obtained from the roots of 17 clinically healthy first premolars from young individuals undergoing tooth extraction due to orthodontic reasons. Routine dental cleaning including scaling and polishing was performed before teeth extraction. Patients were advised to brush their teeth thoroughly; dental plaques were removed by teeth repolishing immediately before teeth extraction.

\section{Isolation, culture, and preparation of stem cells}

Preparation and drape, and local anesthesia were performed. There are several procedures to minimize the risk of transmitted infection: rinsing patient's teeth by diluted povidine-iodine, sterilizing instruments precisely, washing sterile gloves with normal saline and wearing mask and surgical gown. After extraction, the coronal portion of root was held by a sterile pliers and the crown was separated by a disk. Copious amount of saline was used for rinsing in order to reduce temperature and avoid cell damage. Cells should not be damaged by inadvertent movements of pliers tip. The root was immediately entered into tube containing Hanks' Balanced Salt Solution (HBSS) medium and tube was covered and sealed by wax sheet. Only periodontal ligament tissues attached to the middle third of the root surface were scraped with surgical scalpel.

The stem cells culture was conducted according to Gay et al. as follows (14). PDL cells were scraped from premolars and enzymatically digested for $1 \mathrm{~h}$ at $37{ }^{\circ} \mathrm{C}$ in a solution of $3 \mathrm{mg} / \mathrm{ml}$ collagenase type I (Sigma Co., Germany). Then the samples were centrifuged at $400 \times \mathrm{g}$ for $10 \mathrm{~min}$. PDL cells were grown in suspension culture at $37{ }^{\circ} \mathrm{C}$ in $5 \%$ humidified $\mathrm{CO} 2$ in $\alpha$-MEM (Minimum Essential Medium Eagle- Alpha Modification) (Gibco Co., Germany) containing $15 \%$ FBS (Gibco Co., Germany), $100 \mathrm{U} / \mathrm{ml}$ penicillin- $\mathrm{G}, 100 \mu \mathrm{g} / \mathrm{ml}$ streptomycin, and $0.25 \mu \mathrm{g} / \mathrm{ml}$ fungizone (Gibco Limited, Uxbridge, United Kingdom) in a 6 well plate as primary culture. When the cells reached 80 $90 \%$ confluence, cultures were washed twice with phosphate buffer saline (PBS) and the cells were trypsinized with $0.25 \%$ trypsin in $1 \mathrm{mM}$ EDTA (Gibco, Germany). At day 7, cells reached 80-90\% confluence, adherent cells were centrifuged and resuspended in serum supplemented medium in tissue culture polystyrene flasks at a density of $5 \times 10^{3}$ cells $/ \mathrm{cm}^{2}$.

\section{Flow cytometry}

The second passage of the cells were used for flow cytometry analysis following incubation with primary FITC or PE conjugated monoclonal antibodies (CD90, CD105, CD146, CD31, CD34, CD45 Ab) (R\&D Systems Co., China) and then adding fluorescein-conjugated secondary antibody. The cells were washed twice and fixed with 4\% paraformaldehyde. The cell surface markers (CD90, CD105, CD146 positive and CD31, CD34, CD45 negative cells) were analyzed and sorted using a FACSCalibur flow cytometer (Becton Dickinson) by collecting 10,000 events.

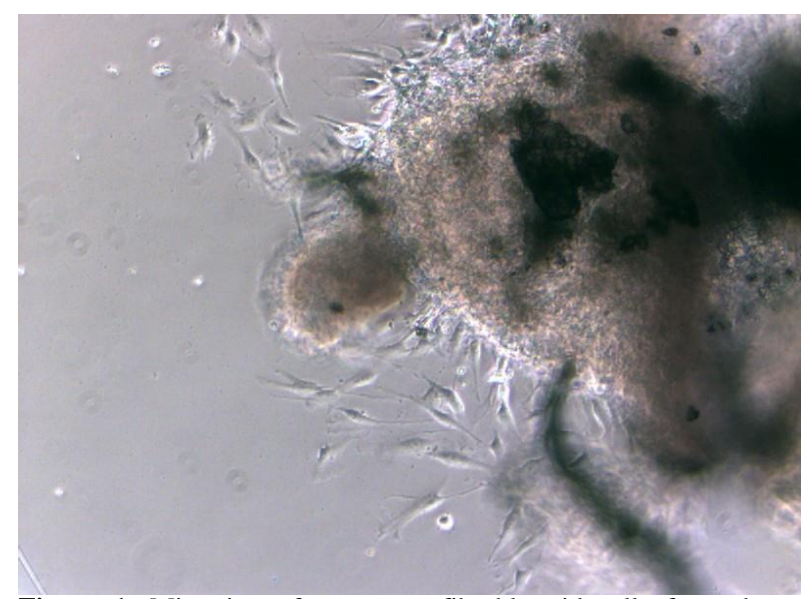

Figure 1. Migration of numerous fibroblastoid cells from the explants after 3 days of culture. 


\section{Osteogenic differentiation}

Adherent cells obtained from second passage wereused for osteogenic differentiation. The cells wereplated in tissue culture dishes in osteogenic medium,including modified minimum essential medium ( $\alpha$-MEM) supplemented with $10 \%$ FBS, $10^{-8} \mathrm{M}(0.01 \mu \mathrm{M})$ Dex (Iran Hormone Pharmaceutical Co, Iran), $5 \mathrm{mM} \beta$-glycerophosphate and $50 \mu \mathrm{g} / \mathrm{ml} \mathrm{1-}$ ascorbic acid.

Cultures in $\alpha$-MEM containing $10 \%$ FBS without osteogenic supplements were considered as control. The cells were then refed every 3 days until calcified nodule-like structures were observed (usually after 4 weeks). These nodular aggregates of cells were morphologically evaluated by inverted microscope and subsequently prepared for histochemical analysis.

\section{Histochemical Analysis}

The presence of calcified nodules and mineralization were confirmed using Alizarin Red-S staining. After rinsing the cells twice with PBS, ice-cold 70\% ethanol was added to fix the cells. Then the cells were washed with deionized water and stained with $40 \mathrm{mM}$ Alizarin Red-S (pH 4.2) (Sigma-Aldrich, Germany) for at least $10 \mathrm{~min}$ at $37^{\circ} \mathrm{C}$. Non-specific stain was removed by washing with PBS. The plates were then air dried and visualized under inverted microscope. The calcium content was measured using o-cresolphthalein complex method at 7, 14, 21, and 28 days of subculture according to the manufacturer's instruction (Pars Azmun, Iran).

Moreover, osteoblastic differentiation was assessed by measuring alkaline phosphatase (ALP) activity in the culture medium using alkaline phosphatase assay kit (Pars Azmun, Iran). Each assay was repeated three times and the mean value of the results was recorded.

\section{Statistical analysis}

Statistical analysis was performed using SPSS version 17. The statistical method was selected according to verification of normal distribution of varibles using Kolmogorov-Smirnov test. Difference in continuous variables in two groups was measured by Student $t$ test. One-way analysis of variance (ANOVA) and the Tukey's post hoc were used to compare the differences between more than 2 groups. P-value less than 0.05 was considered as statistical significance.

\section{Results}

In this study, a pluripotent stem cell population derived from human PDL. At the bottom of the plates the visible cells were mesenchymal type stem cell, adherent spindle shaped and fibroblast-like cells. Primary cultures of PDLSCs mainly consisted of colonies of bipolar fibroblastoid cells. After subcultivation, the cells were proliferated with a population-doubling time of $48 \mathrm{~h}$ and reached a confluent growth-arrested state. After three days of culture, numerous fibroblastoid cells migrated from the explants (Figure 1). Flow cytometry analysis revealed that the cells were positive for CD90, CD105 and CD146 and negative for CD31, CD34, and CD45 as other PDLSCs. After seven days 80$90 \%$ confluent cells were passaged and passage 2 was seeded for differentiation (Figure 2).

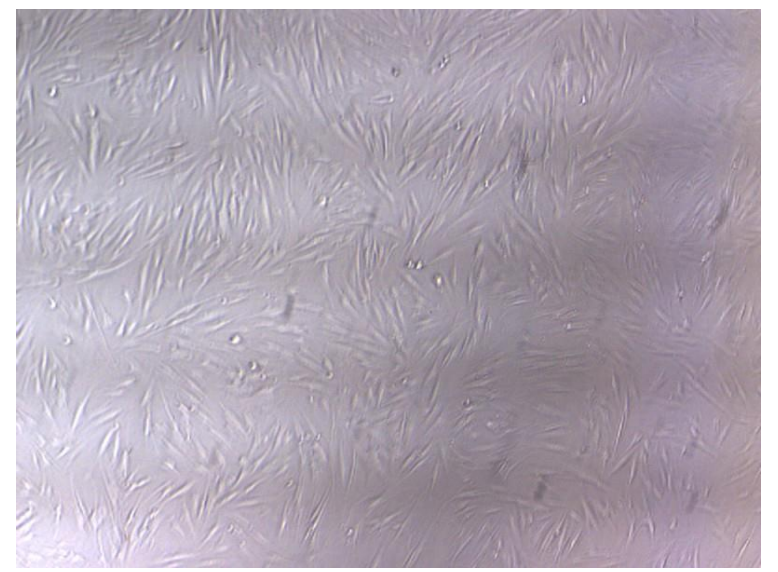

Figure 2. Developing and growing adherent cell layer used for osteogenic differentiation

Morphological analysis of PDLSCs showed oateoblast differentiation (Figure 3).

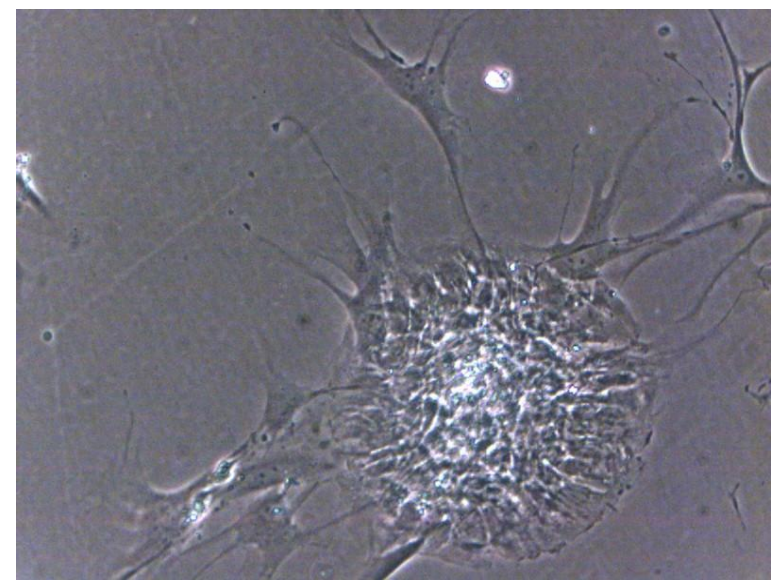

Figure 3. The presence of living cells organized in calcified nodule-like structures.

The results showed the presence of living cells organized in calcified nodule-like structures. The nodular aggregates of cells in differentiating cultures stained with Alizarin Red-S demonstrated that the deposits observed are calcified. Extensive osteogenic differentiation was observed only in PDL-MSCs exposed to osteogenic conditions. 
This is indicated by calcium deposition (Figure 4). While there is no mineralization in the cells cultured in control medium (Figure 5). On the other hand, the highest mineralization was observed on 28th day in Dex-treated PDLSCs compared to what has been found in control group $(480 \pm 62$ vs. $76 \pm 16 \mu \mathrm{M} / \mathrm{ml}$, $\mathrm{p}<0.0001$ ) (Figure 5). The lowest amount of intracellular calcium deposition was observed in control group. An increasing trend of calcium deposition was observed only in Dex treated PDLSCs (Figure 6). On the $7^{\text {th }}$ day, ALP activity of PDLSCs was different in Dex group compared to that of the control group but this difference was not statistically significant. Interestingly, ALP activity increased significantly on $14^{\text {th }}$ day in Dex group compared to that of the control group $(620 \pm 66$ vs. $28 \pm 14 \mathrm{U} / \mathrm{L}$, $\mathrm{P}<0.0001$ ) (Figure 7).

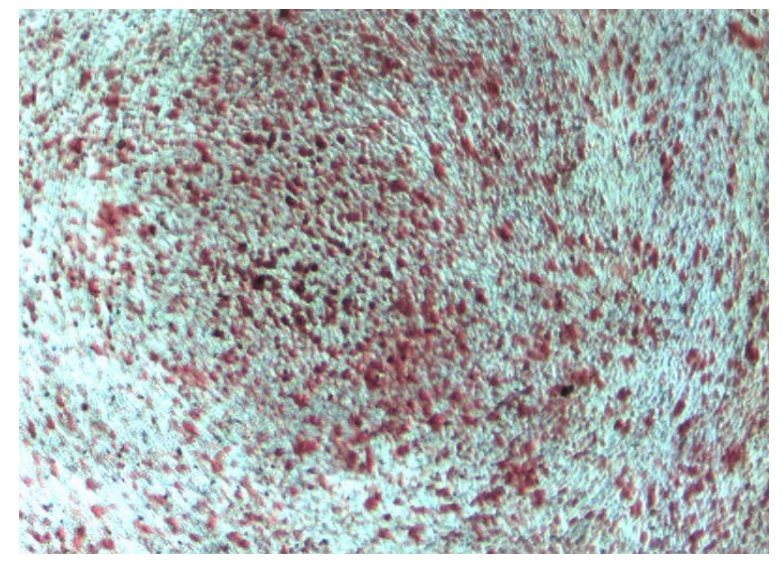

Figure 4. The deposits observed in calcified nodule-like structures stained with Alizarin Red-S which indicates calcium deposition

\section{Discussion}

The aim of the present study was to investigate the capability of human periodontal ligament stem cells for osteogenic differentiation. Numerous biological and clinical studies provide the evidence that PDL are capable of differentiating into calcified-forming osteoblasts under certain conditions. The first human dental stem cells were obtained from dental pulp tissue of extracted third-molar teeth that are able to form a dentin/pulp-like complex. They share similar features with bone marrow mesenchymal stem cells (BMSCs). The dental pulp stem cells (DPSCs) produced dense calcified nodules and did not form adipocytes, whereas BMSCs were calcified throughout adherent cell layer with adipocytes (15). The first isolation of periodontal ligament stem cells occurred in 2004 by Seo et al. They isolated PDLSCs from surgically extracted human third molars. They found that PDLSCs can express mesenchymal stem cells marker such as STRO-1 and CD146/MUC18. Moreover, PDLSCs are able to differentiate into cementoblast-like cells, adipocytes, and collagenforming cells under certain culture conditions. The in vivo analysis on immunocompromised rodents confirmed the PDLSCs potential to produce a cementum/PDL-like structure and involved in periodontal tissue repairing. Therefore, PDL could be considered as a therapeutic strategy for periodontal diseases-derived destroyed tissues (16).

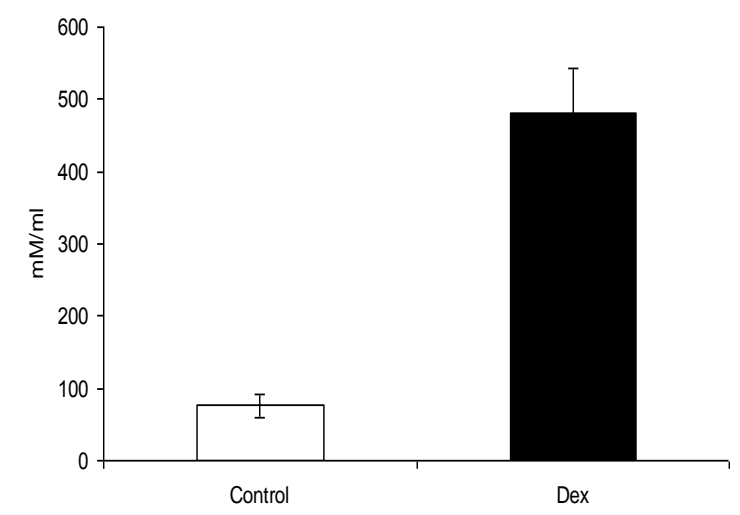

Figure 5. Quantitative analysis of Alizarin Red staining of PDLSCs

The ability of osteogenic differentiation from PDLSCs was previously discussed (17). In our analysis, osteogenic differentiation was induced by culture medium containing ascorbic acid which induces differentiation.

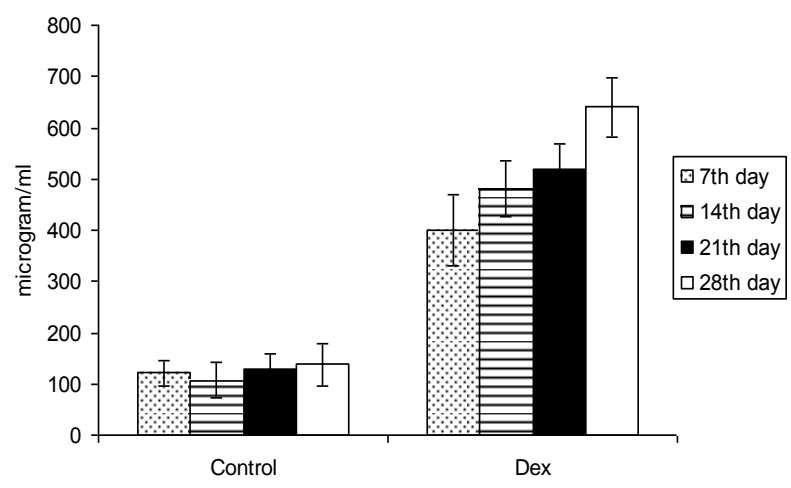

Figure 6. Kinetic pattern of intracellular calcium deposition of PDLSCs under two condition media in $7,14,21$, and 28 weeks.

Several studies demonstrated the osteogenic differentiation from periodontal tissue by different factors and mediators: IL-6 may act as an osteolytic factor and mediate osteoblastic differentiation from periodontal ligament cells (18), this form of differentiation could be induced by simvastatin which inhibits mevalonate pathway and result in human PDL cells proliferation and osteogenic differentiation (19). The impact of icariin on promotion of human 
periodontal ligament cells (hPDLCs) differentiation into osteoblast was confirmed by high osteogenesis gene expression in a study performed by Ding et al (20). The osteogenic differentiation was observed in our study under culture condition treated with Dex. There is some evidence that used Dex for inducing osteogenesis in stem cells. Dex is an osteogenic inducer that are involved in osteoblastic differentiation in human clonal bone marrow cell line (21).

Dex can stimulate the Runx2 (Runt-Related Transcription Factor 2) via $\beta$-catenin-dependent pathway, TAZ (transcriptional co-activator with PDZ-binding motif), and MKP-1 (mitogen-activated protein kinase (MAPK) phosphatase) activation (22). Runx 2 participates in bone formation, regulation of osteoblast differentiation, and leads to increased osteogenic gene expression (23). It was found that Dex is an important factor for mineralization of human mesenchymal stem cells (hMSCs), therefore, hMSCs can act as a candidate source of osteogenesis under optimum culture condition (24). The osteogenic differentiation ability of PDLSCs and pulp of human exfoliated deciduous teeth (SHED) was analyzed by Chadipiralla et al (25). They demonstrated the osteogenic ability of PDLSCs and SHED in medium supplemented with Dex and their potential for in vivo bone regeneration. Thus PDLSCs have a favor capability in clinical and therapeutic applications.

The main necessity of periodontitis treatment is mineralized periodontal tissue reconstruction and its regeneration (26), therefore, osteogenic differentiation seems to be a critical achievement. The importance of PDLSCs regeneration and osteogenic differentiation were demonstrated in many studies.

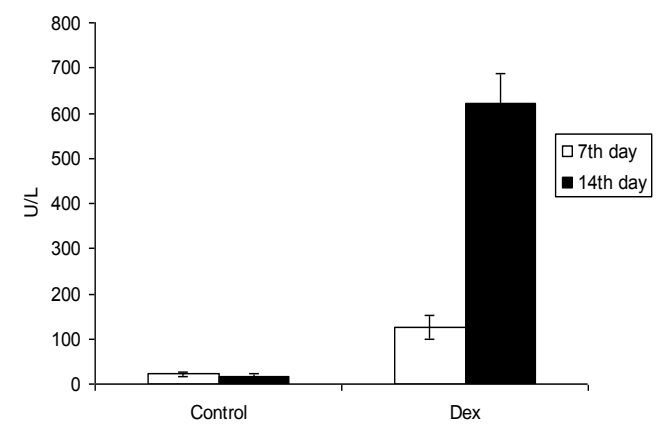

Figure 7. Alkaline phosphatase activity in supernatant of PDLSCs cultures on $7^{\text {th }}$ and $14^{\text {th }}$ days

Encapsulated form of PDLSCs was applied for tendon regeneration after 4 weeks differentiation. High expression of tendon regeneration-relating markers (Scx, DCn, Tnmd, and Bgy) confirm their positive effect. The consistent results derived from in vivo analysis made them as a therapeutic source for tendon engineering (27). IL-10, an anti-inflammatory cytokine, involves in bone mass maintaining and regulating osteoblast production. Thus, IL-10 could be of great benefit in bone loss diseases therapy (28). There are some compounds such as NELL1 that can induce osteogenic differentiation of hPDLSCs, thus, they are associated with periodontal regeneration gene therapy (29).

In summary, the above findings suggest that human PDLSCs have osteogenic differentiation ability and has direct effect on periodontal-relating diseases. Alizarin Red-S staining proved that mineralization occurred in the cell layers during osteoblast differentiation from PDLSCs in cultures containing osteogenic medium. Our study comes to same conclusion as others in which human periodontal ligament tissue-derived MSCs are able to differentiate into the calcified structure and/or osteoblastic lineage. Therefore, PDLSCs could be considered as a gold candidate for periodontal regeneration and its associated diseases.

\section{Conflict of Interest}

The authors declare that they have no conflict of interest in this work.

\section{Authors' Contributions}

HB and MA designed the study, MT analyzed data and reviewed the manuscript. RA and OA performed the experiment and wrote the first draft of the manuscript. MA and HB contributed to experimental design, interpreted data and reviewed the manuscript. All authors reviewed and approved the final version of the manuscript

\section{References}

1. Benatti BB, Silvério KG, Casati MZ, Sallum EA, Nociti FH Jr. Inflammatory and bone-related genes are modulated by aging in human periodontal ligament cells. Cytokine. 2009; 46(2):176-81. PMID: 19251432

2. Ye G, Li C, Xiang X, Chen C, R, Yang X, Yu X, et al. Bone Morphogenetic Protein-9 Induces PDLSCs Osteogenic Differentiation through the ERK and p38 Signal Pathways. Int J Med Sci. 2014; 11(10):1065-72. PMID: 25136261

3. Signat B, Roques C, Poulet P, Duffaut D. Fusobacterium nucleatum in periodontal health and disease. Curr Issues Mol Biol. 2011; 13(2):25-36. PMID: 21220789

4. Spahr A, Klein E, Khuseyinova N, Boeckh C, Muche R, Kunze $\mathrm{M}$, et al. Periodontal infections and coronary heart disease: role of periodontal bacteria and importance of total pathogen burden in the Coronary Event and Periodontal Disease (CORODONT) study. Arch Intern Med. 2006; 166(5):554-9. PMID: 1653404

5. Pihlstrom BL, Michalowicz BS, Johnson NW. "Periodontal diseases". The Lancet, 2005; 366(9499): 1809-1820. PMID: 16298220 
6. Yang H, Aprecio RM, Zhou X, Wang Q, Zhang W, Ding Y, Li Y. Therapeutic Effect of TSG-6 Engineered iPSC-Derived MSCs on Experimental Periodontitis in Rats: A Pilot Study. PLoS One. 2014; 9(6): e100285. PMID: 24979372

7. Sethi M, Dua A, Dodwad V. Stem cell: A window to regenerative density. Int J Pharm Biomed Res. 2012; 3(3): 175180.

8. Melcher AH. On the repair potential of periodontal tissues. J Periodontol. 1976; 47:256-60. PMID: 775048

9. LeBlanc ARH, Reisz RR. Periodontal Ligament, Cementum, and Alveolar Bone in the Oldest Herbivorous Tetrapods, and Their Evolutionary Significance. PLoS One. 2013; 8(9): e74697. PMID: 24023957

10. Hasegawa T, Chosa N, Asakawa T, Yoshimura Y, Asakawa A, Ishisaki A, et al. Effect of fibroblast growth factor-2 on periodontal ligament cells derived from human deciduous teeth in vitro. Exp Ther Med. 2010; 1(2): 337-41. PMID: 22993547

11. Hamilton DW, Oates CJ, Hasanzade A, Mittler S. Migration of periodontal ligament fibroblasts on nanometric topographical patterns: Influence of filopodia and focal adhesions on contact guidance. PLoS One. 2010; 5(12): e15129. PMID: 21152020

12. Bartold PM, McCulloch CA, Narayanan AS, Pitaru S. Tissue engineering: a new paradigm for periodontal regeneration based on molecular and cell biology. Periodontol 2000. 2000; 24: 253-69. PMID: 11276871

13. Huang CY, Pelaez D, Dominguez-Bendala J, Garcia-Godoy F, Cheung HS. Plasticity of stem cells derived from adult periodontal ligament. Regen Med. 2009; 4(6):809-21. PMID: 19903001

14. Gay IC, Chen S, MacDo ugall M. Isolation and characterization of multipotent human periodontal ligament stem cells. Orthod Craniofacial Res. 2007; 10(3): 149-60. PMID: 17651131

15. Gronthos S, Mankani M, Brahim J, Robey PG, Shi S. Postnatal human dental pulp stem cells (DPSCs) in vitro and in vivo. Proc Natl Acad Sci USA. 2000; 97(25): 13625-30. PMID: 11087820

16. Seo BM, Miura M, Gronthos S, Bartold PM, Batouli S, Brahim $\mathrm{J}$, et al. Investigation of multipotent postnatal stem cells from human periodontal ligament. Lancet. 2004; 364(9429): 149-55. PMID: 15246727

17. Li Y, Li SQ, Gao YM, Li J, Zhang B. Crucial role of Notch signaling in osteogenic differentiation of periodontal ligament stem cells in osteoporotic rats. Cell Biol Int. 2014; 38(6):729-36. PMID: 24677709

18. Iwasaki K, Komaki M, Mimori K, Leon E, Izumi Y, Ishikawa I. IL-6 induces osteoblastic differentiation of periodontal ligament cells. J Dent Res. 2008; 87(10):937-42. PMID: 18809747
19. Yazawa H, Zimmermann B, Asami Y, Bernimoulin JP. Simvastatin promotes cell metabolism, proliferation, and osteoblastic differentiation in human periodontal ligament cells. J Periodontol. 2005; 76(2):295-302. PMID: 15974856

20. Ding Q, Zhang FQ, Ma YS. Effect of icariin on osteoblastic differentiation gene expression of human periodontal ligament cells. Beijing Da Xue Xue Bao. 2013; 45(6):975-8. PMID: 24343085

21. Ogston N, Harrison AJ, Cheung HF, Ashton BA, Hampson G. Dexamethasone and retinoic acid differentially regulate growth and differentiation in an immortalised human clonal bone marrow stromal cell line with osteoblastic characteristics. Steroids. 2002; 67(11):895-906. PMID: 12234625

22. Langenbach F, Handschel J. Effects of dexamethasone, ascorbic acid and $\beta$-glycerophosphate on the osteogenic differentiation of stem cells in vitro. Stem Cell Res Ther. 2013; 4(5):117. PMID: 24073831

23. Zhang YY, Li X, Qian SW, Guo L, Huang HY, He Q, Liu Y, et al. Down-regulation of type I Runx2 mediated by dexamethasone is required for 3T3-L1 adiopogenesis. Mol Endocrinol. 2012; 26(5):798-808. PMID: 22422618

24. Mostafa NZ, Fitzsimmons R, Major PW, Adesida A, Jomha $\mathrm{N}$, Jiang $\mathrm{H}$, et al. Osteogenic differentiation of human mesenchymal stem cells cultured with dexamethasone, vitamin D3, basic fibroblast growth factor, and bone morphogenetic protein-2. Connect Tissue Res. 2012; 53(2):117-31. PMID: 21966879

25. Chadipiralla K, Yochim JM, Bahuleyan B, Huang CY, GarciaGodoy F, Murray PE, Stelnicki EJ. Osteogenic differentiation of stem cells derived from human periodontal ligaments and pulp of human exfoliated deciduous teeth. Cell Tissue Res. 2010; 340(2):323-33. PMID: 20309582

26. Rodriguez-Lozano FJ, Insausti CL, Iniesta F. et al. Mesenchymal dental stem cells in regenerative dentistry. Med Oral Patol Oral Cir Bucal. 2012; 17:e1062-7. PMID: 22926467

27 Moshaverinia A, Xu X, Chen C, Ansari S, Zadeh HH, Snead ML, et al. Application of stem cells derived from the periodontal ligament or gingival tissue sources for tendon tissue regeneration. Biomaterials. 2014; 35(9):2642-50. PMID: 24397989

28. Zhang Q, Chen B, Yan F, et al. Interleukin-10 Inhibits Bone Resorption: A Potential Therapeutic Strategy in Periodontitis and Other Bone Loss Diseases. Biomed Res Int. 2014; 2014:284836. PMID: 24696846

29. Chen CY, Liu YJ, Shi SG, Chen FM, Cai C, Li B, et al. Osteogenic differentiation of human periodontal ligament stem cells expressing lentiviral NEL-like protein 1. Int $\mathbf{J}$ Mol Med. 2012; 30(4): 863-9. PMID: 22767336 